\title{
Interdisciplinary Development of a Daily Use Caregiver Sleep Survey (DUCSS): A Mixed Methods Design
}

Jennifer Hughes, Tanvi Banerjee, William Romine, Garrett Goodman, Abby Edwards, and Larry Lawhorne

Wright State University

\section{Acknowledgments}

We thank members of the Wright State Physicians Department of Geriatrics for their support. We also acknowledge the following students for their assistance on this project: Morgan Freeman, social work; and JoAnna Evans, social work.

\section{Funding}

This work was supported in part by the National Institutes of Health [grant K01 LM012439-02].

\section{Author Note}

Correspondence concerning this article should be addressed to Jennifer Hughes, Wright State University, 275 Millett Hall, 3640 Colonel Glenn Hwy, Dayton, OH 454350001. Email: Jennifer.hughes@,wright.edu

\section{Conflict of Interest}

The authors declare that there is no conflict of interest. Institutional Review Board (IRB) provided approval for this research. 


\begin{abstract}
Caregiving for dementia-related illness could result in caregiver health concerns, such as depression, stress, and sleep disturbance. A daily use caregiver sleep survey (DUCSS) was developed using a mixed method design to evaluate burden. A focus group evaluated the survey. The tool was distributed to 24 caregivers and validated using the Rasch statistical model, which indicated that the 15-item survey produced sleep quality measures of sufficient reliability for both group-level and individual-level comparisons (reliability $=0.87$. The sample size was sufficient to provide precise measures of item difficulty (reliability $=0.85$ ), so outcomes associated with levels of sleep quality could be evaluated. We observed that the instrument's structure is unidimensional, which means that the wording of the items does not contain systematic biases peripheral to sleep quality. DUCSS is a useful tool to incorporate into caregiver assessment and monitoring systems.
\end{abstract}

Keywords: caregivers, dementia, daily use sleep survey, Caregiver Assessment using Serious Gaming Technology (CAST) 
By 2025, the number of individuals with Alzheimer's-related dementia is projected to reach more than 7 million. Care for individuals with dementia falls upon loved ones nearly $85 \%$ of the time. Over 16 million Americans provide 18.4 billion hours of unpaid care per year, valued at over 200 billion dollars (Alzheimer's Association, 2018).

Providing care for an individual with dementia is more challenging than caring for those suffering from other illnesses (Ory, Hoffman, Yee, Tennstedt, \& Schultz, 1999). Individuals living with dementia require increasing supervision and frequently display behavioral and emotional changes, such as losses in judgment or communication difficulties. This progressive path is challenging for caregivers, who are, in turn, at risk of stress, depression, financial difficulties, and new or worsened health conditions (Alzheimer's Association, 2018). Assisting the caregiver benefits not only the caregiver, but also the care receiver and society in general.

Caregiver assistance is an important area for expansion. In particular, there is tremendous potential for growth in technology to improve quality of life and future outcomes for caregivers (Adler \& Mehta, 2014; Brown et al., 2019). Researchers have developed a smartphone application, Caregiver Assessment using Serious Gaming Technology (CAST), for use in assessing stress in dementia caregivers. CAST includes gaming assessment, stress scale development, reminders, participant reward system, and biomarkers (Hughes, Banerjee, \& Lawhorne, 2017). CAST developers identified sleep disturbance as an important component, as providing care to a loved one with dementia can result in poor sleep hygiene (McKibbin et al., 2005). Thus, several health and sleep scales were evaluated and incorporated into the development of DUCSS. 


\section{Conceptual Framework}

In the United States, 15.9 million people are caring for dementia patients. Such care results in physical and psychological illness because of the intense physical, psychological, financial, and familial strain placed on the caregiver. As dementia progresses, the caregiver assumes increasing responsibility and supervision of their loved one, while maintaining their own obligations (Alzheimer's Association, 2018). Caregivers have reported experiencing a reduction in quality of life and increases in stress while completing care tasks (Chiao, Wu, \& Hsiao, 2015). Caregivers also reported a decline in personal health and physical health outcomes, and experienced depression that correlates with caregiver stress (Lu \& Wykle, 2007). Stress and depression are common among caregivers (Mausbach, Chattillion, Roepke, Patterson, \& Grant, 2013), and the caregiver's mental health decline was found to directly correlate with the number of dementia-related behaviors exhibited by their loved ones (Robinson, Crawford, Buckwater, \& Casey, 2016).

Sleep disturbance is a contributing factor to caregiver burden. Rowe, McCrea, Campbell, Pe Benito, and Cheng (2008), found that caregivers slept less, took longer to fall asleep, and experienced greater variability in overall sleep patterns. Caregiver sleep disturbance is a concern, with causes ranging from difficulty falling or staying asleep, to insomnia resulting from stress or depression, to irregular sleeps patterns functioning as coping strategies for time management. Some older adults may be predisposed to sleep difficulties, which are exacerbated by providing care (McCurry, Logsdon, Teri, \& Vitiello, 2007). Women are more prone to sleep difficulties and two-thirds of dementia caregivers are women (Alzheimer's Association, 2018). Poor sleep has physical and 
psychological consequences. Min and Slattum (2016) found that older adults with sleep difficulties were at high risk for falls. Depression may impact a caregiver's experience of sleep and result in sleep disturbance (Pinquart \& Sörensen, 2004). Simpson and Carter (2013) found that the caregiver's subjective sleep quality decreased as the number of the patient's dementia behaviors increased. Peng and Chang (2013) reported that depression, demographics such as gender or age, and characteristics of the care recipient are all factors that influence caregiver sleep. The ability to cope with stressors also contributes to sleep efficiency in dementia caregivers. Conversely, stress management techniques utilized by the caregiver led to better sleep outcomes (Taylor et al., 2015).

Identification of caregiver stress allows for timely intervention, which helps to prevent crisis situations and ultimately lead to better outcomes for the dementia patient (Haley, Levine, Brown, \& Bartolucci, 1987). Individuals with dementia are more likely to be placed in long-term care facilities as caregivers experience burnout (Porter et al., 2016) or experience other difficulties in caregiving (Vugt et al., 2005). Given the relationships among caregiver stress, sleep quality, and patient behaviors, the task of measuring and potentially predicting sleep quality for caregivers becomes beneficial.

The purpose of this study was to validate a sleep survey for use with caregivers of dementia patients and is to be used in conjunction with CAST, which aims to assess changes in functioning related to task performance because a caregiver sleep scale would augment the CAST system. While surveys exist to measure sleep quality, these instruments were unsuitable for this function as they were not intended for daily use, as CAST is. Furthermore, some surveys are designed to measure specific disorders such as sleep apnea, which may not depict the experience of an otherwise healthy caregiver. The 
Calgary Sleep Apnea Quality of Life Index (SAQLI) consists of questions specific to the experience of sleep apnea (Flemons \& Reimer, 1998), making it a poor choice for measuring quality of sleep in healthy individuals. Silva, Goodwin, Vana, and Quan (2016) found that, when compared, there were no significant differences between four well-known sleep surveys. Billings et al. (2014) found no significant differences between the Functional Outcomes of Sleep Questionnaire (FOSQ) (Weaver et al., 1997) and the SAQLI (Flemons \& Reimer, 1998).

Development of a tool that can proactively recognize sleep difficulties may improve quality of life for caregivers (Cupidi et al., 2012). Caregivers who proactively manage sleep hygiene are able to address the needs of their care recipient for longer periods of time, thereby allowing the loved one to remain in their home (Hope, Keene, Gedling, Fairburn, \& Jacoby, 1998).

\section{Method}

\section{Procedure}

This study used a cross-sectional, mixed methods design. To create the survey, aspects of the Pittsburgh Sleep Quality Index (PSQI) (Buysse, Reynolds, Monk, Berman, \& Kupfer, 1989), FOSQ (Weaver et al., 1997), SAQLI (Flemons \& Reimer, 1998), and RAND 36-Item Health Survey (SF-36) (Hays, Sherbourne, \& Manzel, 1993) were reviewed. The PSQI measures aspects of sleep quality and insomnia to provide information to clinicians and patients. This survey was not designed for use with caregivers. Many of the original items were not considered for our caregiver survey; however, items relating to sleep latency — how long an individual takes to fall or go back to sleep-were incorporated. For instance, an item from the PSQI that was included in 
the development of our caregiver survey was "How many hours of sleep did you get last night?" (Buysse et al., 1989). Many questions from the FOSQ, which measures sleep quality in patients with sleep apnea, were found to be appropriate measures of daytime functioning and perceived quality of life in caregivers. Examples of items included on the DUCSS survey included "Do you have difficulty performing employed or volunteer work because you are sleepy or tired?" and "Has your relationship with family or work colleagues been affected because you are sleepy or tired?" (Weaver et al., 1997). The SAQLI, which measures quality of life of sleep apnea patients, provided questions regarding quality of life and daytime functioning. Relevant questions from the SAQLI included "Did you have difficulty staying awake while reading?" and "Did you have difficulty staying awake while watching television?” (Flemons \& Reimer, 1998). From the SF-36, we included questions such as "Did you have a lot of energy?" and "How often have you felt tired?" (Hays et al., 1993).

Once assembled, DUCSS was presented to a focus group, composed of a multidisciplinary geriatric treatment team, who evaluated the survey for content validity. The focus group refined the survey, streamlined its content, and suggested additional items. Table 1 illustrates focus group recommendations for survey development. Following these recommendations, the 23-item DUCSS was tested.

Participants were recruited as a convenience sample, based on participant accessibility and ease (Weiss, 1995), from a gerontology practice $(n=11)$, Greene County Council on Aging Dementia Support Group $(n=10)$, and the Miami Valley Alzheimer's Association $(n=3)$. All participants $(N=24)$ were providing care to a person with dementia. Exclusion criteria included caregivers with moderate cognitive 
impairment. Researchers determined eligibility; obtained written informed consent; asked participants to complete the caregiver sleep survey, which included demographics; and described the care recipient's symptomology, level of functioning, and personal sleep quality (see Table 2). Researchers were available to answer questions and provide information and referral services.

\section{Data Analysis}

We fit the unidimensional Rasch partial credit model (Masters, 1982) to the data using the WINSTEPS software package (Linacre, 2006). The purpose of the Rasch model is to provide a standard for the validity of items, under the assumption that they all measure sleep quality and are independent after accounting for sleep quality. Item validity was evaluated by how well the items fit with the Rasch model, which provides a philosophical data-independent criterion for the validity of survey data. The Rasch model, which specifies that the likelihood of expressing a symptom associated with poor sleep quality is proportional to the difference between the patient's sleep quality and the severity of symptoms, has been used extensively for instrument validation in medical contexts (Johansson, Kottorp, Lee, Gay, \& Lerdal, 2014). The extent to which patients' responses on the survey actually reflect this criterion was assessed through mean-square infit and outfit statistics. These measures of fit have expected values of 1, but Wright and Linacre (1996) suggested that values between 0.5 and 1.5 indicate items are useful for measuring the latent trait of sleep quality. Misfitting items (mean-square fit $>1.5$ ) represent a significant bias in favor of patients with low sleep quality. This contradiction of scale directionality indicates a validity concern with that item (Bond \& Fox, 2015). 
The expectation that the entire scale is unidimensional was tested using principal components analysis on residuals with respect to the Rasch model. Linacre and Tennant (2009) state that if residuals are random, the first eigenvalue should fall below 2. A first eigenvalue above 2 indicates some systematic departure from unidimensionality in the set of items with respect to a latent trait of sleep quality, thus warranting closer inspection of those items.

\section{Findings}

The caregiver sleep survey was presented to a focus group of medical professionals prior being used with caregivers of dementia patients. The focus group was attended by 15 geriatric interdisciplinary team members - geriatricians, internal medicine residents, a family medicine resident, nurse practitioners, social workers, a community health worker, a medical researcher, and a computer scientist. The focus group was presented with the 25-item caregiver specific sleep survey and each question was discussed. Results from the focus group findings are presented in Table 3. Recommendations included (1) removing two questions; (2) combining three questions into one; (3) adding dropdown answer boxes to nine questions; and (4) changing a question from "Did you find it difficult to sleep wearing the E-4 device?" to "Please share any comments about your experience while wearing the E-4 device," with a box to write in a narrative answer. The focus group also discussed time ranges in answers and the order in which questions are posed. Focus group participants were interested in the sleep interval time frames and expressed concern that the time ranges were too wide. One stated, "You might lose specificity because of the broad ranges of time given in the 
answers." Another stated, "Ranges that are too short will be difficult for individuals to quantify, and time in these questions are just a gross estimate of time."

Focus group participants also discussed the question concerning the use of a sleep aid and wondered if the use of over-the-counter or prescription sleep medicine should be specifically answered, or if the use of a continuous positive airway pressure (CPAP) machine would constitute a sleep aid. There was discussion about alcohol as a sleep aid. One participant stated, "People drink to assist with sleep, even though, medically, we know it doesn't help sleep." Researchers decided to leave the question as written for ease of use in the survey tool. The focus group added dropdown answers to specify both overthe-counter sleep aids and prescription sleep aids.

Questions were removed from the survey per the recommendations of the focus group (see Table 3). Wording asking if the individuals woke up feeling rested and refreshed was eliminated; this was a double bind question as it addressed two different items in one question. The survey was adjusted based on the recommendations and then administered to caregivers for statistical survey validation.

\section{Participant Demographics}

Our sample, $N=24$, were caregivers providing unpaid care to a loved one with dementia. The average participant age was 60.5 years range: $31-86$ years). In terms of race, $88 \%$ of participants were Caucasian $(n=21)$ and $12 \%$ reported other or mixed races. The vast majority of participants $(88 \%)$ were female, while $12 \%(n=3)$ were male. Half of the care receivers experienced mild dementia, 30\% experienced moderate dementia, and 20\% experienced severe dementia-related illness. Participants reported a 
moderate perceived difficulty in providing care to their loved one with dementia-related illness.

Caregivers described the care recipient's symptomatology as including inappropriate social behavior, communication difficulties, depression, agitation, incontinence, aggression, and wandering; depression was the most frequently identified symptom ( $60 \%$ of participants reported it as a problematic symptom), followed by communication difficulties (55\%), agitation (50\%), incontinence (50\%), inappropriate social behavior (30\%), aggression (25\%), and wandering (15\%).

\section{DUCSS Analysis}

The Rasch model yielded insight into the usefulness of the revised sleep scale and its 15 items for drawing inferences about sleep quality outcomes in caregivers of patients with dementia. Results of the analysis are illustrated in Figure 1. The scale level found that 15 items produced sleep quality estimates of sufficient reliability for both group-level and individual-level comparisons (person reliability $=0.87$ ). Our sample size was sufficient to provide precise estimates of item difficulty (item reliability $=0.85$ ), so outcomes associated with a level of sleep quality expressed by particular individuals could be evaluated. Finally, we observed that the instrument's structure is unidimensional - that is, the wording of the items do not contain systematic biases peripheral to sleep quality. Principal components analysis on residuals with respect to the Rasch model yielded a first eigenvalue of 1.87 items of variance, which is below the threshold of 2 suggested by simulation models for ordinal data (Linacre \& Tennant, 2009). The data support these 15 items as a pure measure of sleep quality. 
A majority of the 15 items displayed mean-square fit values below 1.5, indicating that they conform to the philosophical expectation put forth by the Rasch model that the likelihood of reporting experiences reflective of quality sleep increases directly with caregiver's sleep quality. Items "Sleep latency" and "How tired were you yesterday?" did not fit well with the Rasch model, with infit values of 2.09 and 1.47 , and outfit values of 3.41 and 1.60 , respectively. The high misfit (2.09 infit and 3.41 outfit) of sleep latency indicates that asking caregivers how long they took to fall asleep is not necessarily reflective of quality of sleep, possibly indicating that other factors are responsible for delaying sleep besides sleep quality itself, or that some caregivers reported higher quality of sleep despite taking a long time to fall asleep. Asking caregivers how tired they were on the previous day (1.47 infit and 1.60 outfit) did not provide the most reliable measure of sleep quality, possibly because there are many reasons a caregiver may feel tired during the day that are not attributable to poor sleep.

Thus far, our analysis has concerned the validity of the scale and the usefulness of particular items toward drawing valid inferences about sleep quality in caregivers. Next, we focus on how poor sleep is expressed with respect to caregiving duties and personal health. The person-item map (see Figure 1) illustrates the hierarchy of caregivers and items on the survey along the sleep quality scale, where a caregiver high on the scale has reported high-quality sleep, and a caregiver low on the scale has reported lower-quality sleep. Since caregiver reports of experiences were coded such that a high score indicates an outcome of higher-quality sleep, items that sit higher on the scale represent those that require better-quality sleep to achieve. Lowest on the scale are items that assess basic personal care, called "activities of daily living" (ADL). These include navigating work, 
school, or volunteer positions (Item 14: "WorkSchool"); the ability to bathe oneself, eat, and dress (Item 12: "BatheEatDress"); and the ability to do chores such as housework, arranging transportation, or talking on the telephone (Item 13: "Housework"). While the Rasch model predicts that all caregivers will report functioning in positions outside the home (indicated by their location above Item 14 on the scale), two of the caregivers were located below Items 12 and 13, indicating that their ability to perform basic self-care is negatively affected by their poor-quality sleep. Caregivers who have difficulty with ADL due to lack of sleep will have difficulty caring for their loved one while maintaining their psychological health (Taylor et al., 2015).

Higher on the scale is the ability to perform caregiving tasks. Four caregivers were located proximally to or below locations of these items on the sleep quality scale, indicating that they found that low-quality sleep interfered with their ability to carry out caregiving duties. The lowest-positioned items within this group were Items 11 ("Does lack of sleep interfere with your personal relationships?") and 15 ("Did you have difficulty performing your caregiving duties because you felt tired?"). Slightly higher on the scale was Item 4 ("Rate how much sleepiness interfered with your ability to complete tasks"). Twenty of the participants (83\%) had positions on the sleep scale above these items, indicating that a majority slept well enough to be able to take care of themselves and their care recipients adequately.

Most participants were able to perform caregiving tasks, although approximately half suffered deterioration in their own psychological health. This is indicated by their positioning below items relating to psychological health on the scale (Figure 1). Only 10 participants had locations on the sleep scale above Item 10 ("Did you wake up feeling 
rested?") and Item 5 (a high rating on one's quality of day). An additional 5 participants had locations above Item 15 ("Did you find yourself feeling sad, angry, or overwhelmed yesterday because you were tired?") and Item 16 (“Did you experience any generalized aching, joint pain, or headaches because you felt tired?").

A high rating on overall sleep quality (Item 3 on Figure 1) provides the demarcation between a generally positive and functional psychological state and the conscious observation of feeling energized. Eight caregivers were positioned above Items 6 ("Rate how tired you felt") and 7 ("How would you rate your energy level yesterday?"), indicating that these caregivers, located at the top of Figure 1, reported a high enough sleep quality to have an energetic outlook on their day.

\section{Discussion}

As described in the meta-analysis conducted by Pinquart and Sörenson (2003), studies comparing caregivers and non-caregivers have highlighted significant differences in symptoms such as depression, self-efficacy (self-perceived ability to perform caregiving tasks), and subjective well-being. These differences were exaggerated when specifically looking at dementia patient caregivers and non-caregivers, a finding that was potentially influenced by demographic features such as age; relationship to caregiver; and gender, since a majority of dementia caregivers are female (Pinquart \& Sörenson, 2003). From deployment and subsequent analysis of DUCSS with dementia caregivers, we identified aspects that affected quality of sleep for our caregiver cohort.

As shown in Figure 1, two caregivers expressed difficulty in performing ADL (such as dressing or bathing). The average participant age was 60.5. Research by Desai, Lentzner, and Weeks (2001) emphasizes the need for assistance in performing ADL 
within the older population. A little over one-third of caregivers nationwide are 65 years old and older (Alzheimer's Association, 2018). Desai et al. (2001) reported that more than $20 \%$ of older adults received inadequate assistance for $\mathrm{ADL}$, such as transferring and eating. This inadequacy is higher for elderly persons, who require more assistance in their daily routine than those needing minimal or no assistance.

Previous research highlights differences in caregiving duties between dementia and non-dementia caregivers. Dementia caregivers spend an additional 4.5 hours per week providing care than their non-dementia counterparts, and a larger percentage of dementia caregivers provided care on every ADL task—such as dressing, toileting, and feeding — than non-dementia caregivers (Ory, Hoffman, Yee, Tennstedt, \& Schulz, 1999). The increased time needed by these caregivers, in addition to having to deal with dementia symptoms such as wandering or aggression, helps explain why so many of our participants expressed difficulty performing caregiving duties $(N=4)$. This is supported by Pinquart et al. (2003), who found lower self-efficacy among dementia caregivers as compared to caregivers for patients with other chronic conditions. In addition to the caregivers' perceived difficulty in performing caregiving tasks, their ability to have personal relationships outside their care receivers also showed a similar response to their ability to perform caregiving tasks - that is, caregivers who reported difficulty in performing caregiving tasks also reported that lack of sleep affected their personal relationships.

Schulz and Sherwood (2008) described caregiving as similar to a chronic stress experience, due to the extended time periods of continued stress interspersed with unpredictable behavior from the care recipients. The key factors affecting the degree of 
stress among caregivers were found to be the patient's physical and cognitive levels, along with the type and intensity of care provided by the caregiver. Figure 1 indicates that more than half of participants $(n=14)$ experienced problems related to their psychological health, as evidenced in their answers to questions about whether they felt rested after waking up and the quality of their previous day. Figure 1 provides an illustration of the Rasch model using the Wright Map. The DUCSS is figuratively illustrated by placing survey items on the same scale as participant ability, which allows for a comparison; this, in turn, illuminates how the survey was measured in relation to how participants answered the survey items. Caring for patients with mild to moderate dementia still had a strong negative effect on their quality of life and overall well-being. We see psychological effects occurring at a much lower level in quality of sleep for caregivers if we include moderate to severe dementia caregivers, i.e. psychologically affecting more caregivers due to increased cognitive impairment of recipients, as well as increased intensity of required caregiving (Schulz \& Sherwood, 2008).

Research groups, including the Australian Institute of Health and Welfare (2007) and Davidson and Almeida (2014), have explored the relationship between caregiving for persons with dementia and adverse effects on weariness, lack of energy, and resentment about caregiving responsibilities. In our study, the energy level-related questions were found to be at the top of the Rasch scale for the DUCSS (see Figure 1), indicating that these factors were affected after the caregivers reported difficulty in performing daily tasks, caregiving duties, and other psychological/health effects. Relatively high quality of sleep is required for caregivers to wake up feeling energetic. This implies that most caregivers do not sleep well enough to report feeling energetic $(n=16)$. 
Brummett et al. (2006) demonstrated that caregiving negatively affects sleep quality. This study used data modeling techniques such as structural equations to derive relations from survey data on caregivers. Other factors affecting poor sleep quality were lack of social support and negative outlook indicated by measures of perceived stress, depression, and hopelessness. We report similar measures affecting quality of sleep using the Wright Map on the DUCSS scale, providing additional hierarchy for the effect these measures had on caregiver quality of sleep (see Figure 1).

\section{Limitations}

This tool was developed to use in conjunction with a smartphone application that evaluates dementia caregivers stress and burden. While this survey measures sleep quality, there may be other sources of poor sleep quality that were not considered in the survey, such as addition issues or other physiological sleep health concerns.

Relationships with the care receiver can contribute to caregiver stress; specifically, caregivers with poor relationships with their receivers can have higher levels of stress and burden, which could then exacerbate quality of life and sleep (McCurry et al., 2007). The care receiver's nocturnal and daily behavior pattern could provide additional insight into the caregivers' daily duties, stress, and burden. Quality of sleep is crucial in order for caregivers to sustain caregiving tasks. The care recipient's sleep disturbances are frequently cited as reasons for transitioning a family member into an alternative care facility (Hope et al., 1998). In general, care receiver behavior can affect caregiver sleep. Evaluating quality of sleep in caregivers can play a pivotal role in early interventions such as allaying caregiver burden with professional help, facilitating access to resources, or connecting them to community resources. 


\section{Conclusions and Implications}

The survey provides insight into sleep disturbances of caregivers and their connection with daily quality of life by establishing a hierarchy of functionality using the DUCSS model. Daily living forms the lowest tier, followed by caregiving tasks, and topped by psychological well-being. Developing and understanding the hierarchy is useful in predicting potential, future sleep-related symptomology of caregivers. Further research is needed to refine this questionnaire. Here, we worked with a small convenience sample of dementia-related caregivers. While convenience sampling includes a reduction in scientific precision and potentially biases the research (Weiss, 1995), it is the least disruptive testing format in challenging circumstances, such as that of the dementia caregivers. Future research should test this instrument with a larger number of caregivers. This research is beneficial in determining the relationships among caregiver sleep issues, caregiver stress and burden, and caregiver behaviors-as well as evaluating the predictability and utility of DUCSS for caregiver assessment. 


\section{References}

Adler, R., \& Mehta, R. (2014). Catalyzing technology to support family caregiving. Retrieved from http://www.caregiving.org/wpcontent/uploads/2010/01/Catalyzing-Technology-to-Support-FamilyCaregiving_FINAL.pdf

Alzheimer's Association. (2018). 2018 Alzheimer's disease facts and figures. Retrieved from https://www.alz.org/media/HomeOffice/Facts\%20and\%20Figures/facts-andfigures.pdf

Australian Institute of Health and Welfare. (2007). Dementia in Australia:

National data analysis and development. Retrieved from https://www.aihw.gov.au/getmedia/bb7f4ad4-9879-4ce7-8b6bfa3calca3866/dandad.pdf.aspx?inline=true

Billings, M. E., Rosen, C. L., Auckley, D., Benca, R., Foldvary-Schaefer, N., Iber, C., .. . Kapur, V. K. (2014). Psychometric performance and responsiveness of the functional outcomes of sleep questionnaire and sleep apnea quality of life instrument in a randomized trial: the HomePAP study. Sleep, 37(12), 2017-2024. doi:10.5665/sleep.4262

Bond, T. G., \& Fox, C. M. (2015). Applying the Rasch model: Fundamental measurement in the human science ( $3^{\text {rd }}$ ed.) New York, NY: Routledge/Taylor \& Francis Group.

Brown, E. L., Ruggiano, N., Li, J., Clarke, P. J., Kay, E. S., \& Hristidis, V. (2019). Smartphone-based health technologies for dementia care: Opportunities, 
challenges, and current practices. Journal of Applied Gerontology, 38(1):73-81. doi: $10.1177 / 0733464817723088$

Brummett, B. H., Babyak, M. A., Siegler, I. C., Vitaliano, P. P., Ballard, E. L., Gwyther, L. P., \& Williams, R. B. (2006). Associations among perceptions of social support, negative affect, and quality of sleep in caregivers and noncaregivers. Health Psychology, 25(2), 220-225. doi:10.1037/0278-6133.25.2.220

Buysse, D. J., Reynolds, C. F., Monk, T. H., Berman, S. R., \& Kupfer, D. J. (1989). The Pittsburgh Sleep Quality Index: A new instrument for psychiatric practice and research. Psychiatry Research, 28(2), 193-213. doi:10.1016/0165-1781(89)90047-4

Chiao, C. Y., Wu, H. S., \& Hsiao, C. Y. (2015). Caregiver burden for informal caregivers of patients with dementia: A systematic review. International Nursing Review, 62(3), 340-350. doi:10.1111/inr.12194

Cupidi, C., Realmuto, S., Lo Coco, G., Cinturino, A., Talamanca, S., Arnao, V., . . . Lo Coco, D. (2010). Sleep quality in caregivers of patients with Alzheimer's disease and Parkinson's disease and its relationship to quality of life. International Psychogeriatrics, 24(11), 1827-1835. doi: 10.1017/S1041610212001032

Davidson, J. W., \& Almeida, R. A. (2014). An exploratory study of the impact of group singing activities on lucidity, energy, focus, mood and relaxation for persons with dementia and their caregivers. Psychology of Well-Being, 4, 24. doi:10.1186/s13612-014-0024-5

Desai, M. M., Lentzner, H. R., \& Weeks, J. D. (2001). Unmet need for personal assistance with activities of daily living among older adults. The Gerontologist, 
41(1), 82-88. doi:10.1093/geront/41.1.82

Flemons, W. W., \& Reimer, M. A. (1998). Development of a disease-specific healthrelated quality of life questionnaire for sleep apnea. American Journal of Respiratory and Critical Care Medicine, 158(2), 494-503.

Haley, W. E., Levine, E. G., Brown, S. L., \& Bartolucci, A. A. (1987). Stress, appraisal, coping, and social support as predictors of adaptational outcome among dementia caregivers, Psychology and Aging, 2(4), 323-330. doi: 10.1037/08827974.2.4.323

Hays, R. D., Sherbourne, C. D., \& Mazel, R. M. (1993). The RAND 36-Item Health Survey 1.0. Health Economics, 2(3), 2217-2227. doi:10.1002/hec.4730020305

Hope, T., Keene, J., Gedling, K., Fairburn, C. G., \& Jacoby, R. (1998). Predictors of institutionalization for people with dementia living at home with a carer. International Journal of Geriatric Psychiatry, 13(10), 682-690.

Hughes, J. C., Banerjee, T., Goodman, G., \& Lawhorne, L. (2017). A preliminary qualitative analysis of the feasibility of using gaming technology in caregiver assessment. Journal of Technology in Human Services, 35(2), 183-198. doi:10.1080/15228835.2017.1347553

Johansson, S., Kottorp, A., Lee, K. A., Gay, C. L., \& Lerdal, A. (2014). Can the Fatigue Severity Scale 7-item version be used across different patient populations as a generic fatigue measure - a comparative study using a Rasch model approach. Health and Quality of Life Outcomes, 12, 24. doi:10.1186/1477-7525-12-24

Linacre, J. M. (2006). A user's guide to Winsteps: Rasch-model computer programs. Chicago, IL: Winsteps. 
Linacre, J. M., \& Tennant, A. (2009). More about critical eigenvalue sizes (variances) in standardized-residual principal components analysis (PCA). Rasch Measurement Transactions, 23(3), 1228.

Lu, Y, F., \& Wykle, M. (2007). Relationships between caregiver stress and self-care behaviors in response to symptoms. Clinical Nursing Research, 16(1), 29-43. doi: $10.1177 / 1054773806295238$

Masters, G. N. (1982). A Rasch model for partial credit scoring. Psychometrika, 47(2), 149-174. doi:10.1007/BF02296272

Mausbach, B. T., Chattillion, E. A., Roepke, S. K., Patterson, T., \& Grant, I. (2013). A comparison of psychosocial outcomes in elderly Alzheimer's caregivers and non-caregivers. American Journal of Geriatric Psychiatry, 21(1), 5-13. doi:10.1016/japg.2012.10.001

McCurry, S. M., Logsdon, R. G., Teri, L., \& Vitiello, M. V. (2007). Sleep disturbances in caregivers of persons with dementia: Contributing factors and treatment implications. Sleep Medicine Reviews, 11(2), 143-153. doi:10.1016/j.smrv.2006.09.002

McKibbin, C. L., Ancoli-Israel, S., Dimsdale, J., Archuleta, C., von Kanel, R., Mills, P., . . . Grant, I. (2005). Sleep in spousal caregivers of people with Alzheimer's disease. Sleep, 28(10), 1245-1250. doi:10.1093/sleep/28.10.1245

Min, Y., \& Slattum, P. W. (2016). Poor sleep and risk of falls in community-dwelling older adults: A systematic review. Journal of Applied Gerontology, 37(9):10591084. doi:10.1177/0733464816681149

Ory, M. G., Hoffman, R. R., Yee, J. L., Tennstedt, S., \& Schulz, R. (1999). Prevalence 
and impact of caregiving: A detailed comparison between dementia and nondementia caregivers. Gerontologist, 39(2), 177-185.

doi:10.1093/geront/39.2.177

Peng, H. L., \& Chang, Y. P. (2013). Sleep disturbance in family caregivers of individuals with dementia: A review of the literature. Perspectives in Psychiatric Care, 49(2), 135-146. doi:10.1111/ppc.12005

Pinquart, M., \& Sörensen, S. (2004). Associations of caregiver stressors and uplifts with subjective well-being and depressive mood: A meta-analytic comparison. Aging \& Mental Health, 8(5), 438-449.

Pinquart, M., \& Sörensen, S. (2003). Differences between caregivers and noncaregivers in psychological health and physical health: A meta-analysis. Psychology and aging, 18(2), 250-267. doi:10.1037/0882-7974.18.2.250

Porter, C. N., Miller, M. C., Lane, M., Cornman, C., Sarsour, K., \& Kahle-Worbleski, K. (2016). The influence of caregivers and behavioral and psychological symptoms on nursing home placement of persons with Alzheimer's disease: A matched case-control study. Sage Open Medicine, 4, 2050312116661877. doi:10.1177/20503121166618

Robinson, K. M., Crawford, T. N., Buckwater, K. C., \& Casey, D. A. (2016). Outcomes of a two-component intervention on behavioral symptoms in persons with dementia and system response in their caregivers. Journal of Applied Gerontology, 37(5), 570-594. doi:10.1177/0733464816677549

Rowe, M. A., McCrae, C. S., Campbell, J. M., Pe Benito, A. P., \& Cheng, J. (2008). 
Sleep pattern differences between older adult dementia caregivers and older adult noncaregivers using objective and subjective measures. Journal of Clinical Sleep Medicine, 4(4), 362-369.

Schulz, R., \& Sherwood, P. R. (2008). Physical and mental health effects of family caregiving. Journal of Social Work Education, 44(3), 105-113.

Silva, G. E., Goodwin, J. L., Vana, K., \& Quan, S. F. (2016). Obstructive sleep apnea and quality of life: Comparison of the SAQLI, FOSQ, and SF-36 questionnaires. Southwest Journal of Pulmonary Critical Care, 13(3), 137-149. doi:10.13175/swjpcc082-16

Simpson, C., \& Carter, P. (2013). (2013). Dementia behavioral and psychiatric symptoms: Effect on caregiver's sleep. Journal of Clinical Nursing, 22(1-22), 3042-3052. doi:10.1111/jocn.12127

Taylor, B. J., Irish, L. A., Martire, L. M., Siegle, G. J., Krafty, R. T., Schulz, R., \& Hall, M. H. (2015). Avoidant coping and poor sleep efficiency in dementia caregivers. Psychosomatic Medicine, 77(9), 1050-1057. doi:10.1097/PSY.0000000000000237

Vugt, M. E., Stevens, F., Aalten, P., Lousberg, R., Jaspers, N., \& Verhey, F. R. (2005). A prospective study of the effects of behavioral symptoms on the institutionalization of patients with dementia. International Psychogeriatrics, 17(4), 577-589. doi:10.1017/S1041610205002292

Weaver, T. E., Laizner, A. M., Evans, L. K., Maislin, G., Chugh, D. K., Lyon. K., . . . 
Dinges, D. F. (1997). An instrument to measure functional status outcomes for disorders of excessive sleepiness. Sleep, 20(10), 835-843.

doi:10.1093/sleep/20.10.835

Weiss, R. S. (1995). Learning from strangers: The art and method of qualitative interview studies. New York, NY: The Free Press.

Wright, B. D., \& Linacre, J. M. (1996). Reasonable mean-square fit values. In J. M. Linacre (Ed.), Rasch measurement transactions, part 2. Chicago, IL: MESA Press. 
Rasch Model

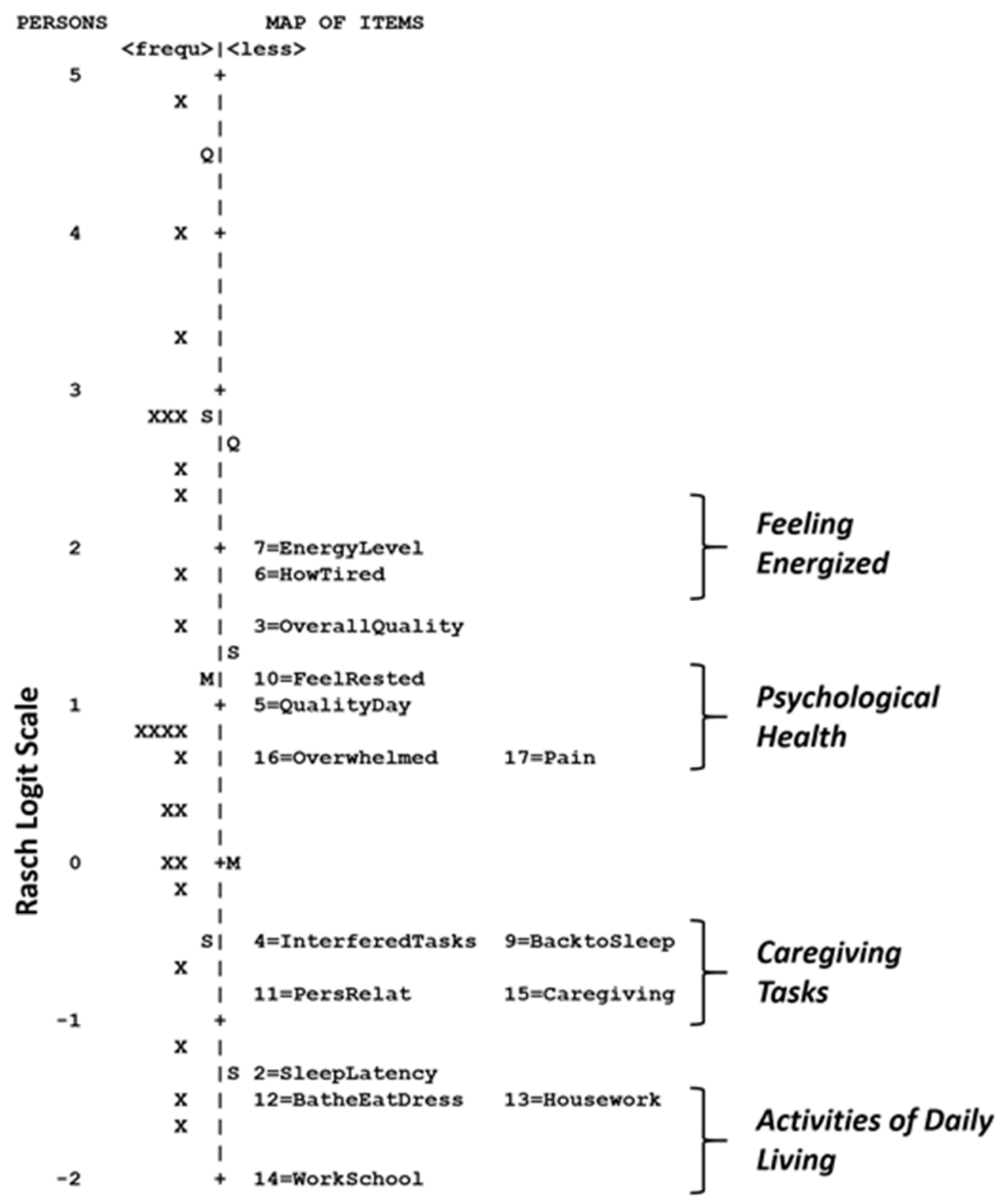

Figure 1. Person-item map of caretakers' measured sleep quality. 
Table 1

Focus Group Recommended Changes from Original Survey to Implemented Tool

Original Survey Question \# $\quad$ Results of Focus Group

$1 . \quad$ No change

2.

Dropdown answer boxes added, added word

"Approximately"

$3 . \quad$ No change

4. No change

5.

Dropdown answer boxes added

6. How much of a problem was it for Question discarded you to stay awake while driving,

eating meals, or engaging in social

activities?

7. How much of a problem was it for Question discarded

you to keep up enthusiasm to get

things done?

8. Dropdown answer boxes added

9. Dropdown answer boxes added

10. Question reworded and turned into written narrative answer

11. Question reworded

12. $\quad$ Words "and refreshed" removed 
13.

14.

15., 16., and 17.

18.

19.

20.

21.

22.

23.

24.

25.
Dropdown answer boxes added, added words "Please check

one"

Dropdown answer boxes added

Added words "Please check one"

Questions combined into one question

Dropdown answer boxes added

Dropdown boxes added, added words "Please check one"

No change

No change

No change

Dropdown answer boxes added, added words "Please check one"

Question reworded 


\section{Table 2}

Summary of Items and Their Scoring Structure on the DUCSS Survey

Survey Question

Scoring

Reverse

Coding?

1: How long did it take to fall asleep?

$<30 \min , 30-60 \mathrm{~min},>\quad \mathrm{N} / \mathrm{A}^{*}$

$60 \mathrm{~min}$

2: Sleep Latency (calculated from Q1)

Ordinal 1-3: $<30 \mathrm{~min}=\quad$ Yes

$1 ; 30-60 \min =2 ;>60$

$\min =3$

3: Rate overall quality of sleep.

Ordinal 1-4

No

4: Rate how much sleepiness interfered with

Ordinal 1-4

Yes your ability to complete tasks.

5: Rate the overall quality of your day $\quad$ Ordinal $1-4 \quad$ No yesterday.
6: Rate how tired you felt.
Ordinal 1-4
Yes

7: How would you rate your energy level

Ordinal 1-4

No yesterday?
8: Did you take a sleep aid last night?
Yes $=1 ;$ No $=0$
$\mathrm{N} / \mathrm{A}^{*}$ 
9: If you woke up during the night how long $\quad$ Ordinal $1-3:<30 \mathrm{~min}=\quad$ Yes did it take to get back to sleep? $\quad 1 ; 30-60 \mathrm{~min}=2 ;>60$

$$
\min =3
$$

$\begin{array}{lll}\text { 10: Did you wake up feeling rested? } & \text { Yes }=1 ; \text { No }=0 & \text { No }\end{array}$

11: Does lack of sleep interfere with personal $\quad$ Yes $=1$; No $=0 \quad$ Yes relationships?

12: Did you find it more difficult to bathe, $\quad$ Yes $=1$; No $=0 \quad$ Yes dress, or eat because you felt tired?

13: Did sleepiness make it difficult to do $\quad$ Yes $=1$; No $=0 \quad$ Yes housework, arrange transportation, or talk on the telephone?

14: Did you have difficulties at work, school, $\quad$ Yes $=1$; No $=0 \quad$ Yes or at a volunteer position due to sleepiness?

15: Did you have a difficulty performing your $\quad$ Yes $=1$; No $=0 \quad$ Yes caregiving duties because you felt tired?

16: Did you find yourself feeling sad, angry, or $\quad$ Yes $=1$; No $=0 \quad$ Yes overwhelmed yesterday because you were tired?

17: Did you experience any generalized

Yes $=1 ;$ No $=0$

Yes 
aching, joint pain, or headaches because you

felt tired?

*Not used directly in the calculation of sleep quality 
Table 3

Rasch Measures and Indices of Fit for Items on the DUCSS Survey

\begin{tabular}{|c|c|c|c|c|}
\hline Item & Measure & SE & Infit & Outfit \\
\hline 2 = Sleep Latency & -1.31 & 0.45 & 2.09 & 3.41 \\
\hline $6=$ How Tired Yesterday & 1.89 & 0.32 & 1.47 & 1.60 \\
\hline $15=$ Caregiving Duties Yesterday & -0.78 & 0.57 & 1.08 & 1.00 \\
\hline $5=$ Quality of Previous Day & 1.07 & 0.35 & 0.92 & 0.93 \\
\hline $17=$ Pain Felt Yesterday & 0.61 & 0.49 & 1.09 & 0.93 \\
\hline $9=$ Time Back to Sleep & -0.49 & 0.37 & 1.48 & 0.92 \\
\hline $3=$ Overall Quality of Sleep & 1.46 & 0.34 & 0.88 & 0.88 \\
\hline $16=$ Felt Overwhelmed Yesterday & 0.64 & 0.50 & 0.78 & 0.80 \\
\hline $7=$ Energy Level Yesterday & 2.07 & 0.38 & 0.60 & 0.61 \\
\hline $11=$ Affected Personal Relationships & -0.79 & 0.57 & 0.94 & 0.56 \\
\hline $10=$ Feel Rested Morning & 1.16 & 0.51 & 0.66 & 0.48 \\
\hline $13=$ Housework Yesterday & -1.55 & 0.67 & 1.01 & 0.47 \\
\hline $4=$ Interfered Caregiving Tasks & -0.49 & 0.33 & 0.59 & 0.44 \\
\hline $12=$ Bathe Eat Dress Yesterday & -1.55 & 0.67 & 0.83 & 0.42 \\
\hline $14=$ Work and School Yesterday & -1.95 & 0.78 & 0.74 & 0.26 \\
\hline
\end{tabular}

